\title{
Anti-recurrent Immunocorrection in Gynecology Andrology and Proctology
}

\author{
Vasyl Beniuk $^{1}$, Vadym Goncharenko ${ }^{1}$, Andrii Kurchenko ${ }^{2}$, Oleksii Tatskyy ${ }^{3}$, Sergii Konovalenko ${ }^{4}$, \\ Svitlana Vintoniuk ${ }^{5}$, Sergii Melnikov ${ }^{6}$, Kamil Nurimanov ${ }^{7}$, Sergii Podpriatov ${ }^{8}$ \\ ${ }^{1}$ Department of Obstetrics and Gynecology, Bogomolets National Medical University, Kyiv, Ukraine \\ ${ }^{2}$ Department of Clinical Immunology, Allergology and Medical Genetics, Bogomolets National Medical University, Kyiv, Ukraine \\ ${ }^{3}$ International Institute of Biotherapy, Kyiv, Ukraine \\ ${ }^{4}$ Department of Monitoring of Tumor Process and Therapy Design, RE Kavetsky Institute of Experimental Pathology, Oncology and \\ Radiobiology, NAS of Ukraine, Kyiv, Ukraine \\ ${ }^{5}$ City Maternity Hospital, Ivano-Frankivsk, Ukraine \\ ${ }^{6}$ Department of Restorative Urology and Innovative Technologies, Institute of Urology AMS of Ukraine, Kyiv, Ukraine \\ ${ }^{7}$ Department of Sexology and Andrology, Institute of Urology AMS of Ukraine, Kyiv, Ukraine \\ ${ }^{8}$ Center for Electric Welding Surgery and New Surgical Technologies, Kyiv, Ukraine
}

Email address:

servlakon@ukr.net (S. Konovalenko)

\section{To cite this article:}

Vasyl Beniuk, Vadym Goncharenko, Andrii Kurchenko, Oleksii Tatskyy, Sergii Konovalenko, Svitlana Vintoniuk, Sergii Melnikov, Kamil Nurimanov, Sergii Podpriatov. Anti-recurrent Immunocorrection in Gynecology Andrology and Proctology. International Journal of Immunology. Vol. 8, No. 1, 2020, pp. 1-8. doi: 10.11648/j.iji.20200801.11

Received: February 7, 2020 Accepted: February 21, 2020; Published: March 2, 2020

\begin{abstract}
The article provides evidence of clinical experience with the use of Arecur ${ }^{\circledR}$ for the treatment of endocervicitis and dysplasia of the cervix, endometrioma, chronic bacterial prostatitis, and for postoperative rehabilitation in patients after removal of hemorrhoids. Arecur ${ }^{\circledR}$ contains exogenous peptides of natural origin - anti-infective proteins of the group of defensins and RJP-1 and has antibacterial, antiviral, anti-inflammatory and antitumor activity. In the study groups, in which Arecur ${ }^{\circledR}$ was assigned to the patients, were reported significantly better clinical efficacy results. The ability of exogenous peptides to mobilize T-lymphocytes to identify and destroy infected and atypical cells determines the elimination of human papilloma virus and creates the conditions for reducing the risk of malignant cell transformation of cervical canal. Arecur ${ }^{\circledR}$, by enhancing the immune supervision of ectopic endometrial cells, promotes regression of clinical symptoms and prolongation of inter-relapsing intervals in patients with endometriosis. In chronic bacterial prostatitis Arecur ${ }^{\circledR}$ increases the efficiency of etiotropic and symptomatic therapy. After surgical removal of hemorrhoid lesions, Arecur ${ }^{\circledR}$ provides more positive dynamics of rehabilitation, improves patients' quality of life and prevents complications. The obtained results allow us to reasonably recommend Arecur $®$ as a medicine for anti-recurrent immunocorrection in gynecology, andrology and proctology.
\end{abstract}

Keywords: Anti-Recurrent Immunocorrection, Exogenous Peptides, Arecur, Endocervicitis, Dysplasia, Human Papilloma Virus, Chlamydia, Endometrioma, Prostatitis, Hemorrhoids

\section{Introduction}

Peptides, as the primordial matrix of life management, appeared on our planet more than three billion years ago and are integral links of biochemical reactions that provide an adequate response to damage in organism. In researches, it was found that peptides have the most ancient, formed evolutionary properties: protection of cells from harmful factors and an adequate response of immune system to a possible danger. [1, 2] Years of researches have shown, that peptides activate immune cells, set up lymphocytes on destruction of pathogenic bacteria and viruses, stimulate antitumor immunity. The activity of peptides contributes to the formation of a complex set of sequential and cross biological reactions that serve a common goal - the 
restoration of immune homeostasis. [3, 4] In fact, peptides compensate for the lost reserves of the immune system to effectively counteract infectious and inflammatory processes both at the local level and at level of macroorganism. Not only an understanding of these mechanisms, but also a wellconsidered assessment of the potential of their clinical significance, naturally led to the development of drugs based on exogenous peptides.

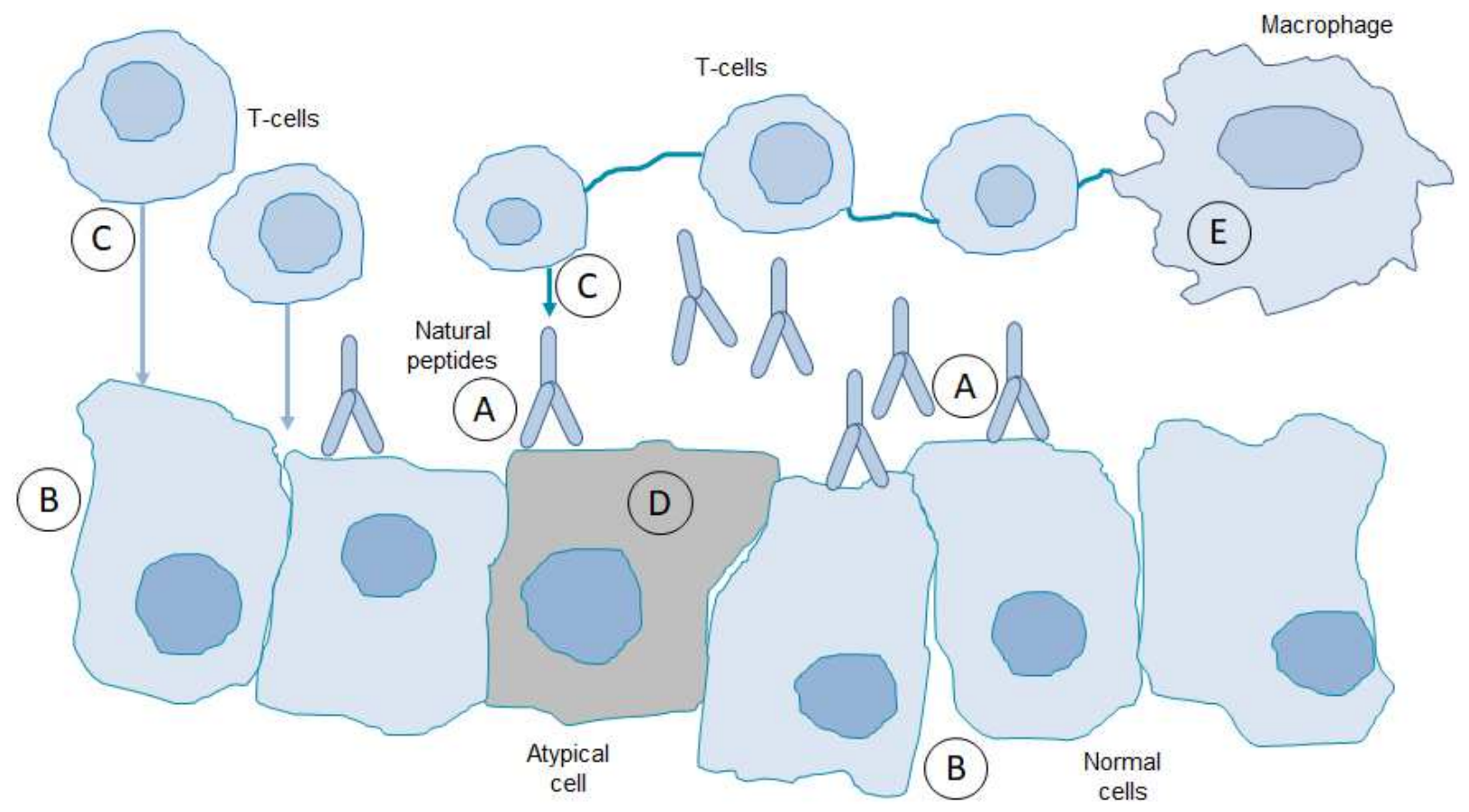

Figure 1. The action of natural exogenous peptides in the target organ.

The interaction is not selective: peptides (A) make contact with all cells (B), irritating their receptors, altering the physicochemical and electrical characteristics of the membranes. These changes do not go unnoticed. The target cells are re-tested with T-lymphocytes (C), thereby increasing the likelihood of detecting an atypical cell (D) and then killing and recycling it with macrophages (E). (Figure 1) [5]

When the patient's body is weakened by chemotherapy or antibiotics, exogenous peptides, by interacting with membrane receptors of lymphocytes and macrophages, enhance the activity of cellular immunity. The action of exogenous peptides in the body is not selective: from one side, these substances, having evolutionarily determined abilities to destroy pathogenic microorganisms, realize a large-scale anti-infection program, and from the other side, attaching to the membranes of target cells, they force $\mathrm{T}$ lymphocytes retest tissues for genetic or infectious lesions, resulting in the identification and destruction of atypical cells. Further, the remnants of the destroyed cells are absorbed by macrophages. In essence, the mechanism of action of exogenous peptides is extremely simple: enable immune cells to detect, destroy and utilize the danger [5-7].

Obtaining from natural sources of biologically active substance, which consists of peptides of the defensin group and RJP, became the foundation for the creation of an innovative anti-relapse immunocorrector, which later received the original name Arecur ${ }^{\circledR}$.

\section{Endocervicitis and Cervical Dysplasia}

The first clinical experience with exogenous peptides was accumulated in gynecology. [5] Based on the first positive results, it was possible to develop new approaches to the complex treatment of cervical dysplasia.

In modern conditions, chlamydia is recognized as a dangerous and socially relevant disease [8]. In fact, mixed infections, including the combination of chlamydia and papillomavirus infection, in almost $100 \%$ of cases cause inflammatory and destructive changes, which lead to infertility and other serious complications. In gynecological practice is known the role of human papillomaviruses, herpes viruses, Epstein-Bar virus in inflammation of the vaginal mucous membranes and cervix, and their influence on the formation of foci of dysplasia and subsequent malignant transformation of epithelial cells has been established. Viral infections in gynecology are crucial etiologic factors for the development of cervical cancer, so their timely diagnosis and comprehensive treatment are important [9-11]

\subsection{Materials and Methods}

In the Department of Obstetrics and gynecology of the Bogomolets NMU, Kyiv, Ukraine, 144 women with cervical dysplasia-II caused by mixed chlamydial-papillomavirus infection were observed. To determine the effect of immunocorrection using exogenous peptides (Arecur ${ }^{\circledR}$ ) on the efficiency of the treatment of dysplasia patients were 
divided into two groups: the main $(\mathrm{n}=72)$ and control $(\mathrm{n}=$ 72) - comparable in age, manifestations and duration of the process. All patients were examined in full, taking into account the anamnesis, social and family status. The examination included gynecological examination, cytological examination of the cervical epithelium, colposcopy, histological examination. To control the efficacy of the proposed treatment, patients in both groups were evaluated for secretory immunoglobulin: $\operatorname{sIgA}$ in the cervical mucus prior to treatment and at the end of treatment 30 days later. For the treatment of pathology of the cervix, a radio-wave electrosurgery apparatus was used, which allowed for biopsy, excision and ablation of abnormally altered tissues on an outpatient basis under local anesthesia.

Patients in the research group received etiotropic antichlamydia therapy - josamycin $500 \mathrm{mg}$ three times a day for 10 days, cone-excision of the pathological focus was performed by radio wave method, as well as cervical biopsy to verify cytological and colposcopic diagnoses. After excision, women were given interferon alfa- $2 b$ in the form of suppositories once daily in the morning and suppositories with chlorhexidine and dexpanthenol - once daily in the evening for 10 days. From the 11th day after excision, the women of the research group received a preparation Arecur as an anti-relapsing immunocorrector: 1 injection intramuscularly 1 time a day in the morning and 1 suppository vaginally 1 time a day in the evening for 20 days. Patients in the control group were treated by a similar scheme, but without the use of immunocorrection.

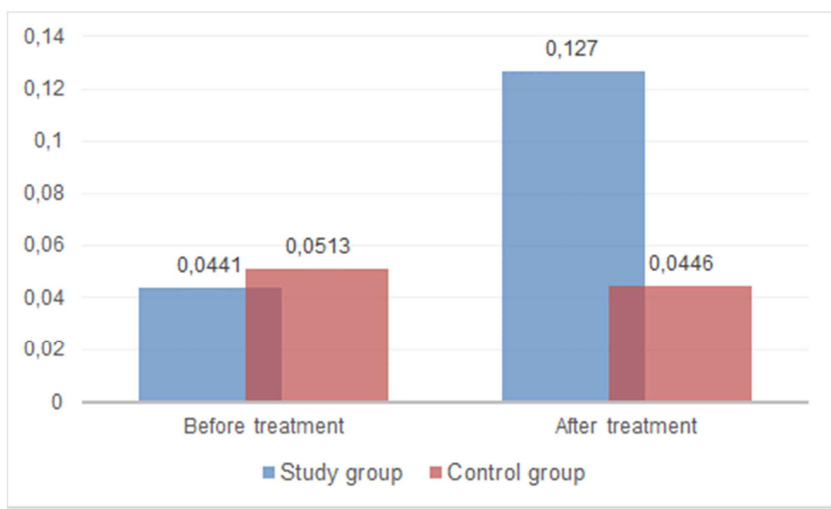

Figure 2. Level of $\operatorname{sg} A(p g / m l)$ in cervical mucus in patients with associated papillomavirus-chlamydial cervical dysplasia.

\subsection{Results}

During the research was found, that the concentration of sIgA in the mucus of the cervical canal in the main and control group, which were comparable $(\mathrm{p}>0.05)$ at the beginning of treatment, subsequently had different dynamics. As a result of the application of the Arecur immunocorrector, the $\operatorname{sIgA}$ concentration increased significantly in the main group from $0.0441 \pm 0.014$ to $0.127 \pm 0.004(\mathrm{p}<0.001)$, whereas in the control, the sIgA concentration did not change significantly (from $0.0513 \pm 0.011$ to $0.0446 \pm 0.003, \mathrm{p}=0.558$ ). After treatment, a significantly higher sIgA concentration than the control was retained in the main group, $\mathrm{p}<0.001$ (Figure 2).
Increased rates of secretory IgA indicate the superiority of the recommended method over the standard method of treatment, because it creates the conditions for the elimination of pathogens. It should be emphasized, that all patients of the compared groups at the beginning of the study by PCR were found to have high and low oncogenic types of human papilloma virus in a clinically significant concentration $(>3.0$ $\mathrm{lg}$ ). During the observation period, the first group compared to the second group showed a more pronounced decrease in the concentration of the virus. Thus, HPV of high cancer risk in the study group at the beginning of the study was $66(91.7 \%)$ cases, at the end of $14(19.4 \%), \Delta=-72.2 \%, \mathrm{p}<0.001$. In the second group, $64(88.9 \%)$ and $25(34.4 \%)$, respectively, $\Delta=-$ $54.2 \%, \mathrm{p}<0.01$. And further, during the 6 months of observation in the study group, we observed a positive trend: the disappearance of clinical manifestations of HPV infection and cervicitis; improvement of the colposcopic picture; normalization of the results of cytological examination; achievement of HPV DNA elimination in scrapings according to real-time PCR data; viral load reduction (quantitative format for HPV determination). Against the backdrop of generally positive results in the study group of papillomavirus infection after 6 months occurred in $2(2.7 \%)$ women, chlamydia - in 6 $(8.3 \%)$, it is not possible to exclude re-infection. Instead, in the comparison group at the end of the observation period, HPV infection was identified in $14(19.4 \%)$ women $(p=0.001)$ and chlamydia in $11(15.3 \%)(\mathrm{p}=0.196)$, which determines the risk of endocervic relapse. and dysplasia in this group is significantly higher.

\subsection{Conclusion}

The results obtained allow us to consider anti-recurrent immunocorrection with the use of the drug Arecur clinically justified and recommend its carrying out in groups of patients with chronic infectious-inflammatory diseases in gynecological practice.

\section{Endometriosis}

The mechanism of action of exogenous peptides of Arecur drug in endometriosis is most likely due to their ability to integrate into the membrane of an ectopically located endometrial cell and attract T-lymphocytes and macrophages to interact with it. The synthesis of TNF- $\alpha$ by immunocompetent cells and, as a consequence, the recruitment of inactive $\mathrm{T}$ lymphocytes in the microenvironment of the endometrioma naturally leads to the initiation of programmed death of ectopic cells, where the caspase system plays the leading role in the cascade. Cells, that have undergone apoptosis, are fragmented and subsequently disposed of by macrophages. [12]

\subsection{Materials and Methods}

In the clinic of the Institute of Urology of the AMS of Ukraine, Kiev, and in City Maternity Hospital, IvanoFrankivsk, Ukraine, endometriosis was treated in 84 women of childbearing age. In 38 patients, uterine endometriosis 
(adenomyosis) was diagnosed, in 20 - endometriosis of the vaginal part of the cervix. In order to study the effeciency of Arecur drug in treating endometriosis, patients were divided into two groups. In the study group $(n=42)$, patients received a combined preparation of progestogen and estrogen (dienogest $2 \mathrm{mg}+$ ethinylestradiol $0.03 \mathrm{mg}$ ) once a day for 180 days, as well as an anti-relapse immunocorrector, the drug Arecur from the first day after discontinuing gestagen therapy, 1 injection intramuscularly 1 time per day in the morning and 1 suppository vaginally 1 time per day in the evening for 20 days. In the comparison group $(n=42)$, women received a combined preparation of progestogen and estrogen (dienogest $2 \mathrm{mg}+$ ethinylestradiol $0.03 \mathrm{mg}$ ) once a day for 180 days without a course of anti-relapse therapy. In patients with a colposcopically confirmed diagnosis of endometriosis of the vaginal part of the cervix, pathological lesions were removed using argon plasma coagulation.

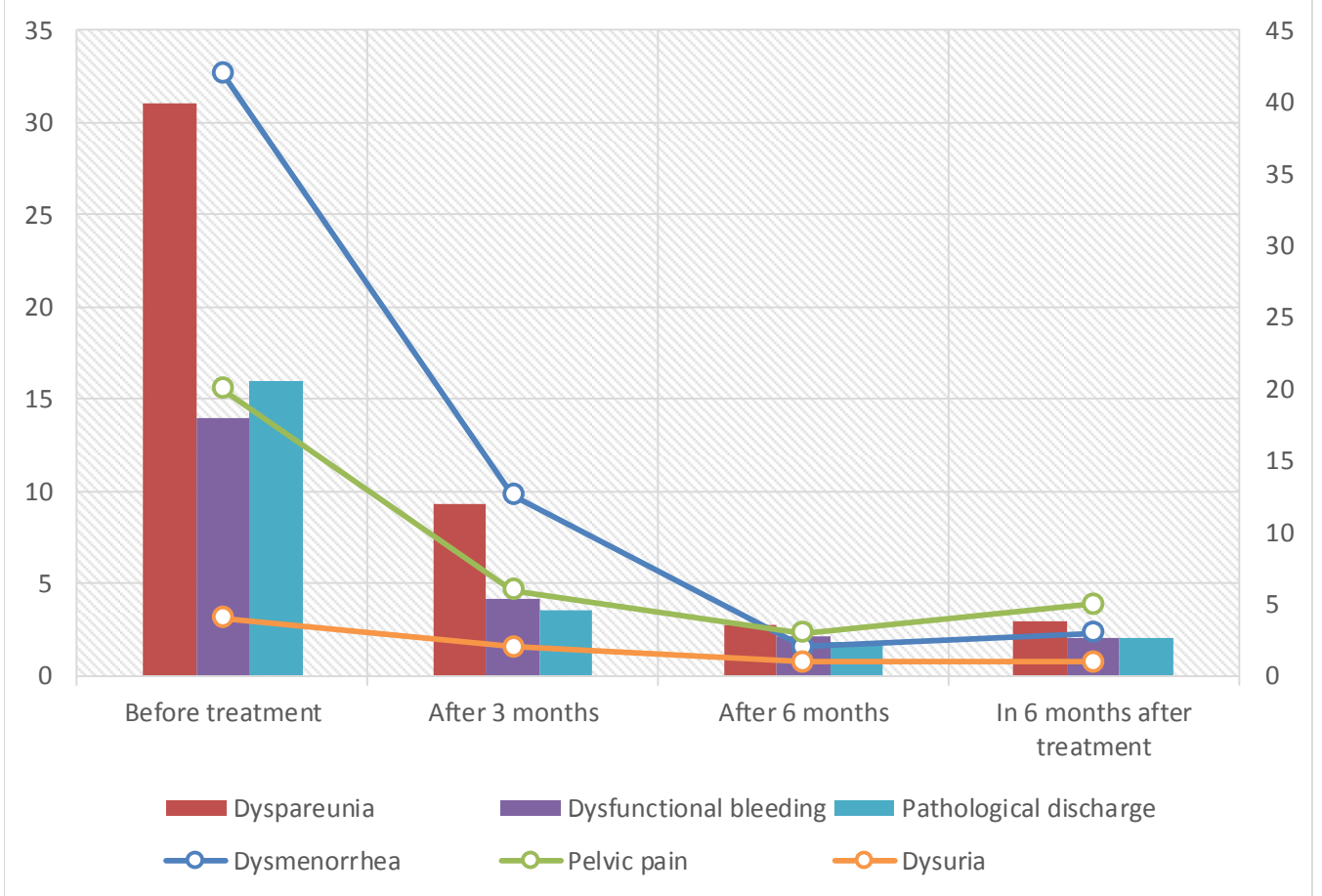

Figure 3. The number of patients with clinical manifestations of endometriosis at different stages of observation in the study group $(N=42)$.

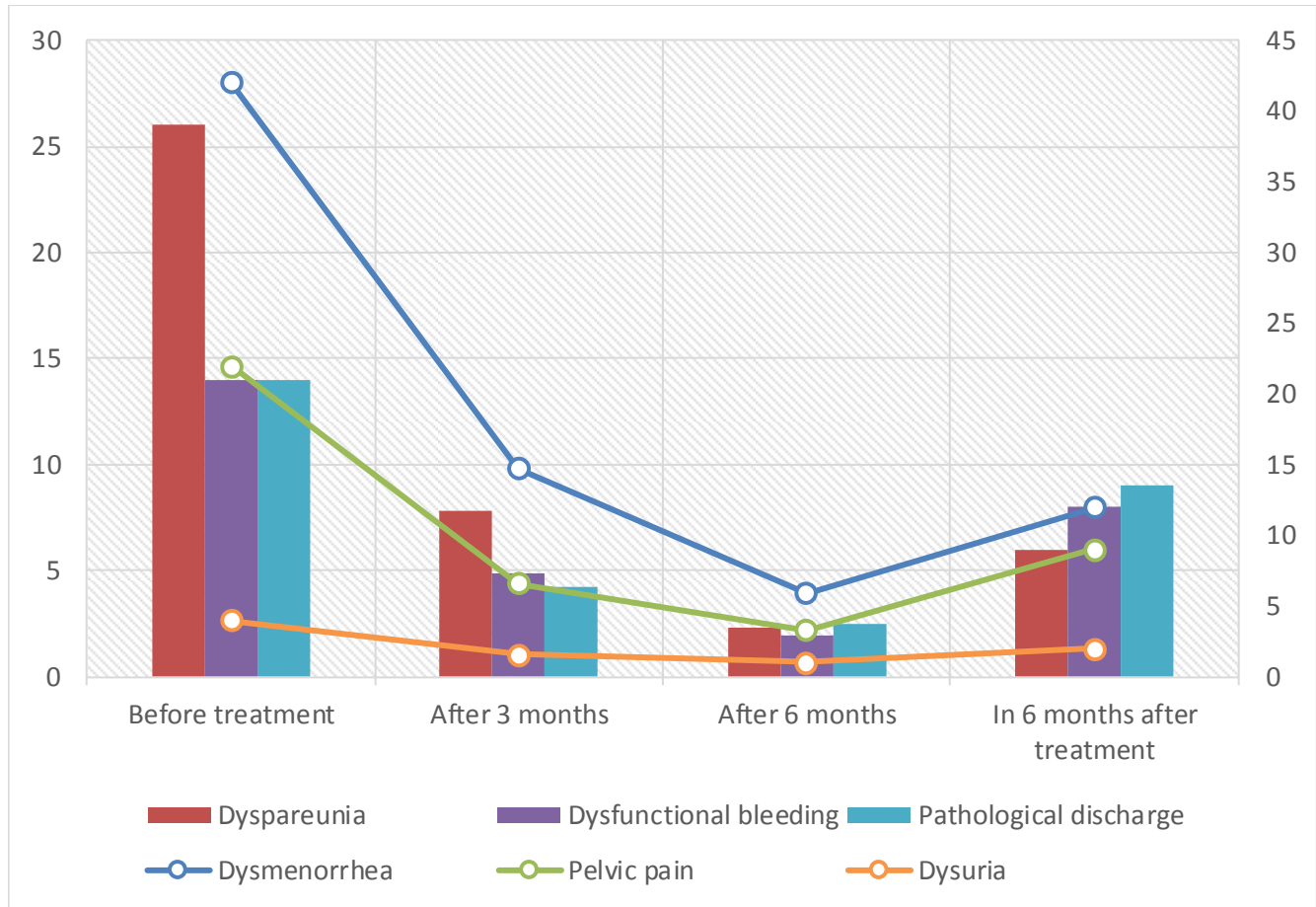

Figure 4. The number of patients with clinical manifestations of endometriosis at different stages of observation in the comparison group $(N=42)$. 
The course of the disease was monitored from the first day of treatment with the previous fixation of symptoms before the start of therapy, after 3 and 6 months, and also 6 months after the end of the course of therapy.

\subsection{Results}

In both observation groups, there was a persistent regression of the clinical symptoms of the disease throughout the entire period of treatment with progestogen and ethinylestradiol (Figures 3, 4). If all women had dysmenorrhea in the study group before treatment, then by the end of the 180-day course of hormone therapy it was fixed in 2; dyspareunia - before treatment in 31 and 3 after, pelvic pain in 20 and 3 , respectively, dysfunctional bleeding in 14 before and after 2, pathological discharge in 16 before and 2 after, dysuria was observed in 4 patients before and 1 after treatment. In total, at the end of the course of hormone therapy, 9 (21.4\%) of 42 women retained some complaints. In the comparison group of these patients, there were 7 $(16.6 \%)(\mathrm{p}=0.579)$.

However, it should be noted that at the next control point 6 months after the end of the hormonal course, in the group in which there was no anti-relapse immunocorrection, the clinical manifestations of endometriosis began to return to women. For example, dysmenorrhea was recorded in 12 $(28.6 \%)$ women, pelvic pain in $9(21.4 \%)$ and pathological discharge in $9(21.4 \%)$ patients. In total, in this group, one or another symptoms of the disease six months after the end of hormone therapy were observed in $20(47.6 \%)$ women, and 11 of them returned the clinical manifestations of endometriosis after a relatively long period of well-being. On the contrary, in the study group - during the indicated period the number of women with clinical manifestations of the disease did not increase significantly: if after hormone therapy there were $9(21.4 \%)$, then after another 6 months there were $10(23.8 \%)$. The relative risk of detecting clinical manifestations 6 months after the end of therapy in the main group was reduced by $65.6 \%$ - OR $=0.344(0.12-0.96), p=$ 0.022

\subsection{Conclusion}

These results speak not only in favor of the inclusion of exogenous peptides in the complex treatment of endometriosis, but also in favor of the modern concept that the ectopic endometrial cell, being essentially a normal cell but in an atypical location, finding itself in a new place, acquires new signs that may contribute to its transformation into a target for active immunocompetent cells [13-15]. Once in the focal point of endometrioma, exogenous peptides become additional attractive structures for T-lymphocytes and macrophages. Thus, the process of endometrioma disintegration can be initiated, including due to complex cascade reactions of the caspase system, leading to apoptosis of ectopic endometrial cells.

\section{Chronic Bacterial Prostatitis}

The experience of using the drug Arecur with chronic bacterial prostatitis was recognized as successful. There is no doubt that chronic inflammation in the prostate gland, which tends to recur several times a year, requires a systematic approach to therapy. This is primarily due to the fact that conditionally pathogenic microorganisms take part in the occurrence of prostatitis, against which it is difficult to choose antibiotics. Also, prostate tissue is sensitive to other damaging factors: malnutrition, alcohol abuse, sedentary lifestyles, irregular and unhealthy sexual relationships. [1618]. The Department of Sexology and Andrology of the Institute of Urology of the AMS of Ukraine, Kiev, has gained experience in the complex treatment of chronic bacterial prostatitis using Arecur.

\subsection{Materials and Methods}

To determine the role of peptide immunocorrection, patients were divided into two groups. The main group in the complex treatment received levofloxacin $500 \mathrm{mg}$ once a day, the total duration of the course of antibiotic therapy was 14 days; in addition, patients received suppositories with indomethacin $100 \mathrm{mg}$ once daily in the morning and tamsulosin $0.4 \mathrm{mg}$ once daily. As an anti-relapse immunocorrector, Arecur was recommended for patients according to the scheme: 1 intramuscular injection 1 time per day in the morning and 1 suppository rectally 1 time per day in the evening for 20 days. Patients in the reference group received similar treatment without the use of an anti-relapse immunocorrector. Clinical and laboratory control was performed before treatment, after 1 week and after treatment - after 4 weeks.

\subsection{Results}

Signs of improvement were noted in both groups of patients within a week after starting therapy. Patients of the study group $(n=60)$ showed a significant decrease and disappearance of symptoms, as well as normalization of the number of leukocytes in the secretion of the prostate, an increase in the maximum volumetric flow rate of urine according to uroflowmetry (from $15.5 \pm 0.8 \mathrm{ml} / \mathrm{s}$ to $17.3 \pm$ $0.9 \mathrm{ml} / \mathrm{s}, \mathrm{p}=0.140$ ). The average score on the NIH-CPSI scale decreased from $42.8 \pm 5.4$ to $22.9 \pm 3.8, \mathrm{p}=0.004$. The prescribed therapy was well tolerated by patients. In patients of the reference group $(n=60)$, a decrease or complete disappearance of complaints was also noted. The maximum volumetric urine flow rate according to uroflowmetry increased from $15.8 \pm 1.0 \mathrm{ml} / \mathrm{s}$ to $17.5 \pm 1.2 \mathrm{ml} / \mathrm{s}, \mathrm{p}=$ 0.374 . The average score on the NIH-CPSI scale decreased from $38.9 \pm 5.9$ to $20.7 \pm 3.7, \mathrm{p}=0.011$. After treatment, during a control bacteriological examination of the prostate secretion of the main group, bacterial pathogens eradication was achieved in $56(93.3 \%)$ patients; in the comparison group, bacteriological negativity was observed in 47 (78.3\%) 
patients, $\mathrm{p}=0.017$. The data obtained indicate that Arecur is implementing an effective anti-infection program.

But most importantly, within a year after the end of treatment in the reference group, $17(28.3 \%)$ patients had relapses of chronic prostatitis, and in 4 of them - twice during the period. At the same time, in the group of patients who received Arecur, only $5(8.3 \%)$ cases of recurrence were noted during the year. The risk of recurrence in the main group was reduced by $87.1 \%$ - OR $=0.229(0.06-0.72), p=0.004$. Obviously, this may be due to the integration of the anti-relapse immunocorrector, the drug of exogenous peptides Arecur, into the management plan of chronic bacterial prostatitis.

\subsection{Conclusion}

The study confirmed that in achieving successful treatment results for chronic bacterial prostatitis, the immunoregulatory component plays a crucial role and contributes to the fullscale functioning of etiotropic and pathogenetic therapeutic factors. Summarizing the results obtained, it can be concluded that anti-relapse immunocorrection creates the conditions for increasing the effectiveness of the treatment of chronic infectious and inflammatory processes of the prostate gland and contributes to achieving long-term remission of the disease for at least 9-12 months.

\section{Clinical Rehabilitation After Removal of Hemorrhoids}

The presence of two groups of peptides in Arecur defensins, which have a pronounced primary anti-infectious activity and royall jelly proteins (RJP-1) with powerful regenerative potential - open up significant prospects for the use of the drug in the healing of wounds and ulcerative defects. The restorative effects of Arecur were first studied in patients with trophic ulcers against the background of chronic venous insufficiency and the results were positive: ulcer healing was achieved in $56.3 \%$ of patients, while in the control group only in $35.7 \%$ [19]. This gave grounds to use Arecur after surgical interventions, in particular in the form of suppositories, as a regenerating agent after surgery in patients with hemorrhoids on the basis of the clinic of the Center for Electric Welding Surgery and New Surgical Technologies, Kiev, Ukraine.

\subsection{Matherials and Methods}

The study group included 30 patients with hemorrhoids of 3-4 stages. After removal of hemorrhoidal lesions, a modern technique of electric welding of soft tissues was used, which allowed to reduce trauma and ensure the full preservation of the functions of the anal sphincter. Arecur suppositories were used on the third day after removal of hemorrhoidal foci 2 times a day for 10 days. In the control group $(n=30)$, Arecur suppositories were not used. It was decided to evaluate the effectiveness of early and late postoperative rehabilitation: patients of both groups filled out a specially designed questionnaire, where they noted the presence or absence of complaints after surgery, on the 5th day, on the 14th day and 2 months after the operation.

\subsection{Results}

The number of patients with complaints at different stages after surgery is shown in tables 1 and 2 . On the 5th day after the removal of hemorrhoidal lesions, the majority of patients in both groups recorded a variety of symptoms associated with the post-traumatic condition of the rectum.

Table 1. Patient complaints at different stages of the postoperative period, study group.

\begin{tabular}{llll}
\hline Symptoms & Day 5 & Day 14 & In 2 months \\
\hline Pain in the anus at rest & $24(80 \%)$ & $2(6.7 \%)^{*}$ & $0(0 \%)^{*}$ \\
Pain in the urge to defecate & $21(70 \%)$ & $4(13.3 \%)^{*}$ & $7(23.3 \%)^{*}$ \\
Pain during defecation & $26(86.7 \%)$ & $2(6.7 \%)^{*}$ & $1(3.3 \%)^{*}$ \\
Pain after defecating & $22(73.3 \%)$ & $1(3.3 \%)^{*}$ & $1(3.3 \%)^{*}$ \\
Anus discomfort at rest & $11(36.7 \%)$ & $2(6.7 \%)^{*}$ & $0(0 \%)^{*}$ \\
Sensation of the sphincter area at rest & $12(40 \%)$ & $0(0 \%)^{*}$ & \\
\hline
\end{tabular}

* - the dynamics is statistically significant for days $5(\mathrm{p}<0.05)$.

Table 2. Patient complaints at different stages of the postoperative period, control group.

\begin{tabular}{llll}
\hline Symptoms & Day 5 & Day 14 & In 2 months \\
\hline Pain in the anus at rest & $26.4(88 \%)$ & $6(20 \%)^{*}$ & $0(0 \%)^{*}$ \\
Pain in the urge to defecate & $23.1(77 \%)$ & $9(30 \%)^{*}$ & $2(6.7 \%)^{*}$ \\
Pain during defecation & $20.8(69.3 \%)$ & $13(43.3 \%)^{*}$ & $4(13.3 \%)^{*}$ \\
Pain after defecating & $17.6(58.7 \%)$ & $9(30 \%)^{*}$ & $3(10 \%)^{*}$ \\
Anus discomfort at rest & $13.2(44 \%)$ & $4(13.3 \%)^{*}$ & $0(0 \%)^{*}$ \\
Sensation of the sphincter area at rest & $14.4(48 \%)$ & $4(13.3 \%)^{*}$ & $0(0 \%)^{*}$ \\
\hline
\end{tabular}

* - the dynamics is statistically significant for days $5(\mathrm{p}<0.05)$.

By day 14, the number of patients with complaints was predictably reduced and reached a minimum 2 months after surgery $(\mathrm{p}<0.001)$. It should be noted that complaints remained in the study group only in $2(6.6 \%)$ patients, and in the control group in $8(26.7 \%)$ patients, $p=0.035$. Proctitis was diagnosed in these patients, which required additional prescription of anti-inflammatory therapy. Relapse of hemorrhoids in patients of both groups during the 18 months of observation was not observed. 


\subsection{Conclusion}

The data obtained speak in favor of the use of Arecur suppositories in patients in the postoperative period after removal of hemorrhoidal lesions. Arecur promotes the effective restoration of the functional ability of the rectum and prevents the occurrence of a chronic inflammatory process, which means it improves the quality of life of patients.

\section{Summary}

The introduction of Arecur ${ }^{\circledR}$ based on natural exogenous peptides into the routine clinical practice of doctors of various specialties can reasonably be considered as an innovative and promising step forward. Successful clinical observations in gynecology, andrology and proctology provide the foundation for the further development of antirelapse immunocorrection.

The ability of Arecur ${ }^{\circledR}$ anti-infectious peptides not only to destroy pathogenic microorganisms on their own, but also to act as intermediaries between bacteria, viruses, fungi and immune cells - it is a key advantage that is realized primarily in the prevention of relapse of an infectious-inflammatory pathology.

The use of Arecur ${ }^{\circledR}$ provides powerful support for antiinfectious and anti-tumor programs, which are performed every second in the organs and tissues of a person. Each repeated testing signal of a T-lymphocyte stimulated by peptides and addressed to target cells - whether it is an epithelium of the cervical canal, an endometrioid implant, epithelial cells in the prostate or in the mucous membrane of the rectum - is a guaranteed increase in the probability of preventing their own threats that constantly arise in the body.

It is obvious, that a cell infected with a virus, damaged by toxins and showing signs of mutation is a biological weapon directed against its own body, as it can become malignant. Only the immune system can cope with this threat, and exogenous peptides as part of the Arecur ${ }^{\circledR}$ anti-relapse immunocorrector help it in this.

\section{Acknowledgements}

The authors express thanks to the International Institute of Biotherapy for support in conducting research.

\section{Conflict of Interests}

The authors declare that they have no conflict of interest.

\section{References}

[1] Sun CQ, Arnold RS, Hsieh CL, Dorin JR, Lian F, Li Z, Petros JA. Discovery and mechanisms of host defense to oncogenesis: targeting the $\beta$-defensin- 1 peptide as a natural tumor inhibitor. Cancer Biol Ther. 2019; 20 (6): 774-786.

[2] Parvy JP, Yu Y, Dostalova A, Kondo S, Kurjan A, Bulet P, Lemaitre B, Vidal M, Cordero JB. The antimicrobial peptide defensin cooperates with tumour necrosis factor to drive tumour cell death in Drosophila. Elife. 2019 Jul 30; 8: e45061.

[3] Fruitwala S, El-Naccache DW, Chang TL. Multifaceted immune functions of human defensins and underlying mechanisms. Semin Cell Dev Biol. 2019 Apr; 88: 163-172.

[4] Cheng, H. Y., Ning, M. X., Chen, D. K., \& Ma, W. T. (2019). Interactions Between the Gut Microbiota and the Host Innate Immune Response Against Pathogens. Frontiers in immunology, 10, 607.

[5] Kurchenko A. I., Beniuk VO, Potebnya GP, Kobis V. L., Tatskyi O. F., Neimark S. O. The role of exogenous peptides in the renewal of a full immune reaction in the conditions of the second immunodeficiency. Women's health. 2017; №1 (117). - P. 89-97.

[6] Shypulin V, Stoliarova O, Konovalenko V, Tatskyy O, Didenko G, Konovalenko S. Study of the Exogenous Peptide Effect on the TGF- $\beta 1$ Expression-A Risk Factor for the Hepatocellular Carcinoma Recurrence. American Journal of Biomedical and Life Sciences. Vol. 7, No. 4, 2019, pp. 73-78.

[7] Litvinenko O. O, Tatskiy O. F, Konovalenko V. F, et al. Breast Cancer Relapse Prevention: Role of Anti-Relapsing Immunocorrection. Cancer Sci Res. 2019; 2 (2); 1-6.

[8] Sachse K, Laroucau K, Riege K, Wehner S, Dilcher M, Creasy HH, Weidmann M, Myers G, Vorimore F, Vicari N, et al. Evidence for the existence of two new members of the family Chlamydiaceae and proposal of Chlamydia avium sp. nov. And Chlamydia gallinacea sp. nov. Syst Appl Microbiol 2014; 37: 79-88.

[9] Williamson A. L. Chattopadhyay K., Hazra A., Dandara C. The combined risks ofreduced or increased function variants in cell death pathway genes differentially influence cervical cancer risk and herpes simplex virus type 2 infection among black Africans and the Mixed Ancestry population of South Africa. BMC Cancer. 2015; 15: 680.

[10] Wright TC, Stoler MH, Behrens CM, Sharma A, Zhang G, Wright TL. Primary cervical cancer screening with human papillomavirus: end of study results from the ATHENA study using HPV as the first-line screening test. Gynecol Oncol. 2015; 136: 189-197.

[11] D. Wohlmeister, D. Barreto Vianna, Vi. Etges Helfer, F. Gimenes, M. Lopes Consolaro, R. Bones Barcellos, M. L. Rossetti, L. Calil, A. Buffon, D. A. Pilger. Association of human papillomavirus and Chlamydia trachomatis with intraepithelial alterations in cervix samples. Mem Inst Oswaldo Cruz. 2016 Feb; 111 (2): 106-113.

[12] Melnikov SM, Tatskyi O. F. A new look at endometriosis: the role of restoring of full immune surveillance over ectopic cells of the endometrium. Women's health. 2017; № 7 (123). - P. 99-107.

[13] Hou XX, Wang XQ, Li DJ. Roles of regulatory T Cells in pathogenesis of endometriosis. Reprod Dev Med 2019; 3: 117-23.

[14] Yang S, Wang H, Li D, Li M. Role of Endometrial Autophagy in Physiological and Pathophysiological Processes. J Cancer. 2019 Jun 9; 10 (15): 3459-3471.

[15] Vallvé-Juanico J, Houshdaran S, Giudice LC. The endometrial immune environment of women with endometriosis. Hum Reprod Update. 2019 Sep 11; 25 (5): 564-591. 
[16] Gorpinchenko I. I., Migov V. G. Treatment of patients with chronic calculous prostatitis using a peptide biostimulator Prostatilen: first results and prospects // Men's Health. - No 2 (45) 2013. - P. 37-40.

[17] Zaseda Y. I., Solomenny RI, Ganushchak A. V. The experience of radical conservative treatment of chronic calculous-fibrous and fibrous-calculous prostatitis // Ukrainian journal "Healthy, gender and psychosomatic medicine." - 2016.-- No. 1 (03). - P. 34-43.
[18] Zaseda Y. I., Tatskyi O. F. The effect of tissue accumulation of exogenous peptides is the key to understanding the mechanisms of harmonization of the immune homeostasis of patients with chronic bacterial prostatitis. Health of the man. 2017. № №1 (60). - P. 51 - 56.

[19] Podpriatov S, Apostolov V, Tatskyy O, Konovalenko S. Konovalenko V. Angioprotective properties of exogenous peptides. Practitioner №1 (8)-2019; 20-24. 